\title{
Increased FGF-23 levels are linked to ineffective erythropoiesis and impaired bone mineralization in myelodysplastic syndromes
}

\author{
Heike Weidner, ${ }^{1}$ Ulrike Baschant, ${ }^{1}$ Franziska Lademann, ${ }^{1}$ Maria G. Ledesma Colunga, ${ }^{1}$ \\ Ekaterina Balaian, ${ }^{2,3}$ Christine Hofbauer, ${ }^{1,4}$ Barbara M. Misof, ${ }^{5}$ Paul Roschger, ${ }^{5}$ Stéphane Blouin, ${ }^{5}$ \\ William C. Richards, ${ }^{6}$ Uwe Platzbecker, ${ }^{2,3}$ Lorenz C. Hofbauer, ${ }^{1,3}$ and Martina Rauner ${ }^{1}$ \\ 'Bone Lab Dresden, Department of Medicine III \& Center for Healthy Aging, and 'Department of Medicine I, Technische \\ Universität Dresden, Dresden, Germany. ${ }^{3}$ German Cancer Consortium (DKTK), partner site Dresden and German Cancer \\ Research Center (DKFZ), Heidelberg, Germany. ${ }^{4}$ Department of Orthopedics and Trauma Surgery, Technische Universität \\ Dresden, Dresden, Germany. ${ }^{5}$ Ludwig Boltzmann Institute of Osteology at the Hanusch Hospital of OEKG and AUVA \\ Trauma Centre Meidling, 1st Medical Department, Hanusch Hospital, Vienna, Austria. ${ }^{6}$ Amgen, Thousand Oaks, \\ California, USA.
}

Myelodysplastic syndromes (MDS) are clonal malignant hematopoietic disorders in the elderly characterized by ineffective hematopoiesis. This is accompanied by an altered bone microenvironment, which contributes to MDS progression and higher bone fragility. The underlying mechanisms remain largely unexplored. Here, we show that myelodysplastic NUP98-HOXD13 (NHD13) transgenic mice display an abnormally high number of osteoblasts, yet a higher fraction of nonmineralized bone, indicating delayed bone mineralization. This was accompanied by high fibroblast growth factor-23 (FGF-23) serum levels, a phosphaturic hormone that inhibits bone mineralization and erythropoiesis. While Fgf23 mRNA expression was low in bone, brain, and kidney of NHD13 mice, its expression was increased in erythroid precursors. Coculturing these precursors with WT osteoblasts induced osteoblast marker gene expression, which was inhibited by blocking FGF-23. Finally, antibody-based neutralization of FGF-23 in myelodysplastic NHD13 mice improved bone mineralization and bone microarchitecture, and it ameliorated anemia. Importantly, higher serum levels of FGF-23 and an elevated amount of nonmineralized bone in patients with MDS validated the findings. C-terminal FGF-23 correlated negatively with hemoglobin levels and positively with the amount of nonmineralized bone. Thus, our study identifies FGF-23 as a link between altered bone structure and ineffective erythropoiesis in MDS with the prospects of a targeted therapeutic intervention.

Authorship note: UP, LCH, and MR contributed equally to this work.

Conflict of interest: WGR was employed by Amgen at the time of study conduct.

Copyright: (c) 2020, American Society for Clinical Investigation.

Submitted: February 11, 2020

Accepted: June 25, 2020

Published: August 6, 2020.

Reference information: /CI Insight. 2020;5(15):e137062.

https://doi.org/10.1172/jici.

insight.137062.

\section{Introduction}

Myelodysplastic syndromes (MDS) belong to the most common malignant hematological disorders in the elderly, increase markedly with age, and result from altered hematopoietic stem cell (HSC) function within the bone marrow. Ineffective hematopoiesis in MDS characterized by dysplastic cells in the bone marrow and the peripheral blood leads to peripheral cytopenias that determine clinical symptoms (1). However, the pathogenesis of MDS is still discussed controversially. While in earlier studies, an altered phenotype of HSC due by cytogenetic and molecular aberrations was considered the main cause for bone marrow failure and transformation into acute myeloid leukemia $(2,3)$, more recently, disturbances of the bone microenvironment, in which HSC reside, have emerged to initiate, maintain, and propagate MDS (4-6).

Bone is a dynamic organ that undergoes a coordinated process of continuous bone remodeling. First, damaged bone is resorbed by HSC-derived osteoclasts. Afterward, osteoblasts deriving from mesenchymal stem cells (MSC) produce a new and nonmineralized bone matrix (osteoid) that is gradually mineralized, thus providing rigidity (7). Bone remodeling and bone mass maintenance are critically affected in MDS, as patients with MDS have a 2-fold higher risk to develop fragility fractures than their age-matched controls (8). 
MSC and their osteogenic progeny appear to be particularly closely linked to MDS. Deletion of Dicer1 in osteogenic cells results in a MDS-like syndrome in mice (4). In addition, highly activated Wnt signaling and TGF- $\beta 1$ cause functional changes in MSC and contribute to the MDS MSC phenotype $(9,10)$. Moreover, transplantation of myelodysplastic HSC into a healthy niche delays the onset of MDS (11), suggesting an important function of osteoblasts and other niche components in the development of MDS. Importantly, MSC derived from patients with MDS show an altered cytogenetic, cell surface marker and epigenetic profile $(10,12-15)$, as well as an impaired proliferation and differentiation capacity in vitro $(5,15-17)$. These alterations in MDS-derived MSC lead to a disruption in the support of hematopoiesis (18), which may further contribute to MDS pathogenesis and a faster tumor progression. Despite the increasing amount of investigations of the myelodysplastic niche, the molecular mechanisms underlying the disturbed osteohematopoietic niche and its pathogenic communication to HSC remain poorly defined.

Therefore, to obtain deeper insights into the bone microenvironment in MDS, we used a well-established mouse model of MDS, the NUP98/HOXD13 (NHD13) transgenic mouse. This mouse overexpresses the NUP98/HOXD13 fusion protein in hematopoietic cells and develops MDS-like features such as peripheral cytopenias and dysplasia in blood as well as bone marrow cells at the age of 4-6 months, which later also transforms into acute myeloid leukemia (19). Recently, we depicted microstructural abnormalities in the bone microenvironment of NHD13 mice (8); however, the underlying cellular and molecular mechanisms were not explored. Using these mice, we show that myelodysplastic mice have a high fraction of nonmineralized bone in the presence of an increased number of osteoblasts, which coincides with high serum levels of fibroblast growth factor-23 (FGF-23), a hormone regulating phosphate homeostasis and bone mineralization $(20,21)$. Interestingly, this factor has also recently been identified as a negative regulator of erythropoiesis (22). Blocking the excess of FGF-23 in myelodysplastic mice not only normalized bone microarchitecture and promote mineralization of osteoid, but also improved erythropoiesis. Importantly, this mechanism is not restricted to mice, as patients with MDS also have increased serum levels of FGF23 and an increased amount of nonmineralized bone. Thus, this study identifies FGF-23 as a driver in the pathogenesis of MDS and proposes it as a therapeutic target with a dual mode of action.

\section{Results}

Myelodysplastic NHD13 mice show high bone formation activity with increased amounts of nonmineralized bone and elevated levels of FGF-23. Our previous study using $\mu \mathrm{CT}$ analysis indicated an altered bone microarchitecture with a lower number of trabeculae in the femur and vertebra of 6-month-old NHD13 mice (8). In this study, we extended our analysis to histological stainings to obtain deeper insights into the cellular events occurring in the bone of myelodysplastic NHD13 mice. The abnormal bone microarchitecture with a lower trabecular number could be confirmed histologically in an independent cohort using the von Kossa/van Gieson staining (Figure 1A). Beyond that in 6-month-old anemic NHD13 mice, a reduced trabecular number was also found in 2-month-old NHD13 mice, which, at this age, show no hematological abnormalities (Figure 1B). Tartrate-resistant acid phosphatase (TRAP) staining was used to determine the osteoclast and osteoblast number per bone perimeter (Figure 1A). The number of TRAP-positive osteoclasts as well as their activity measured by C-terminal telopeptide of type 1 collagen (CTX-1) did not differ between 2-month-old WT and NHD13 mice (Figure 1, C and D). However, the development of MDS reduced the number of osteoclasts, even though the bone resorption marker CTX1 was not altered (Figure 1, C and D). Interestingly, at both time points, the osteoblast number and the bone formation marker procollagen type $1 \mathrm{~N}$-terminal propeptide (P1NP) were increased 2- to 3-fold in the NHD13 mice compared with age-matched WT controls (Figure 1, E and F). The latter is supported by a higher bone formation rate in NHD13 mice (Figure 1G).

Since the excess of osteoblastic bone formation with low to normal numbers of osteoclasts was not associated with an increased bone volume, the amount of nonmineralized bone (osteoid) was analyzed (marked in pink, Figure 1A). Before the onset of MDS, we observed an increase in osteoid volume and width in NHD13 mice. Similar to the 2-month-old NHD13 mice, the osteoid parameters were also increased in 6-month-old NHD13 mice compared with WT controls (Figure 1, H and I). Despite the higher amount of osteoid, the bone mineralization density distribution (BMDD) (characterizing the mineralized bone matrix) in 4-month-old mice was not different between the groups (Supplemental Figure 1, A-C; supplemental material available online with this article; https://doi.org/10.1172/jci.insight.137062DS1), suggesting that once the mineralization has started in the osteoid, mineral accumulation and final level of 
A

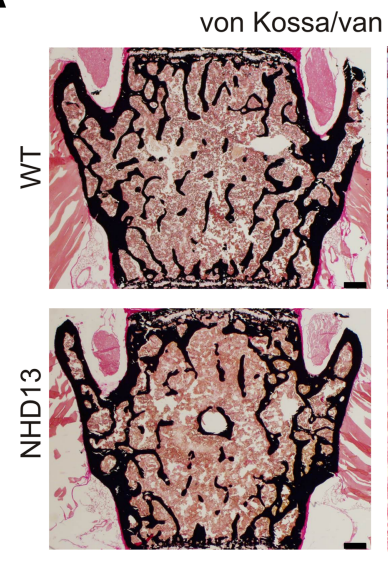

C

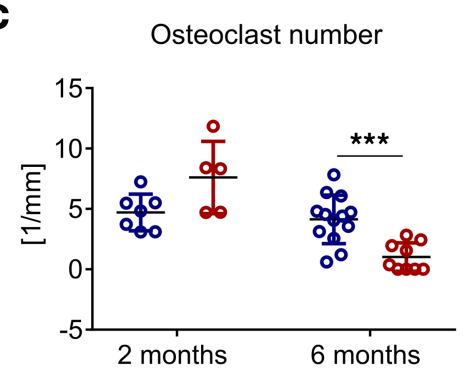

F

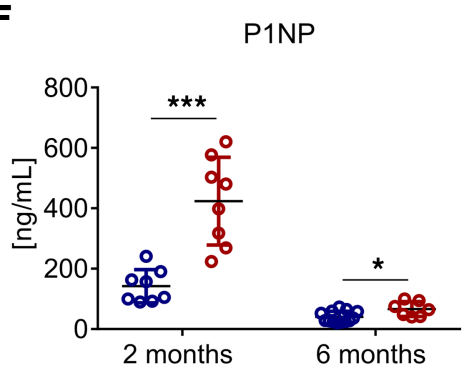

I

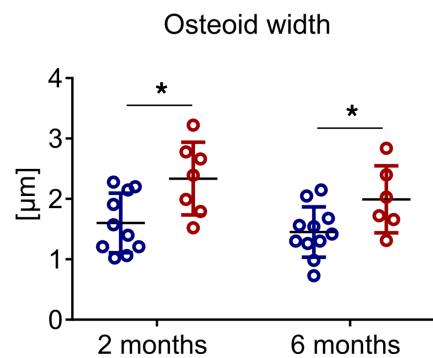

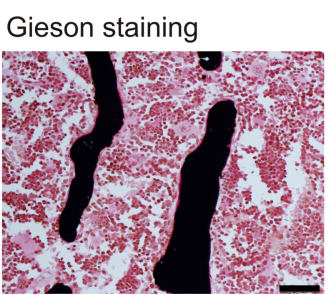

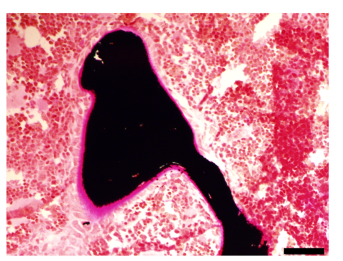

D

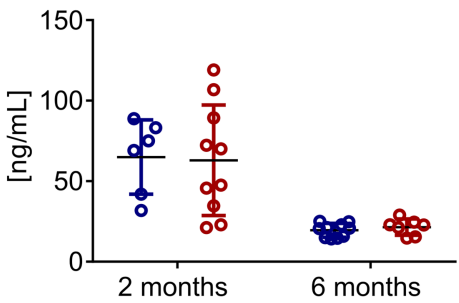

G

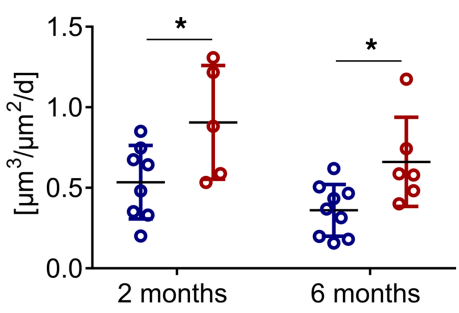

J

C-terminal FGF-23

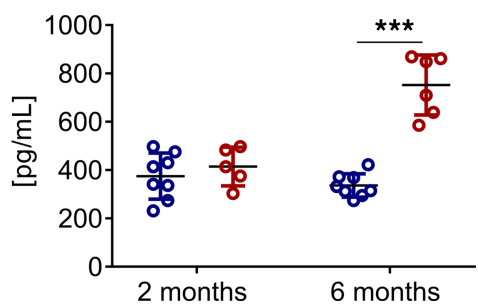

B

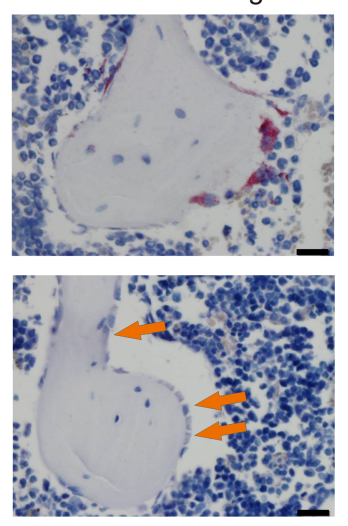

E

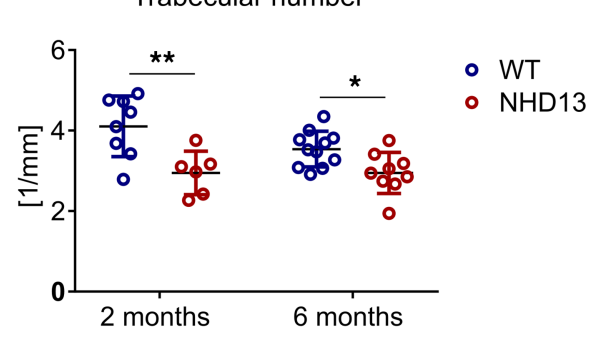

Osteoblast number

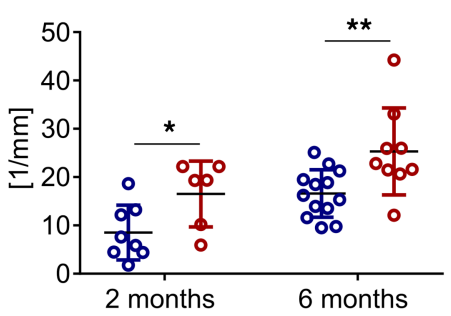

H Osteoid volume/bone volume

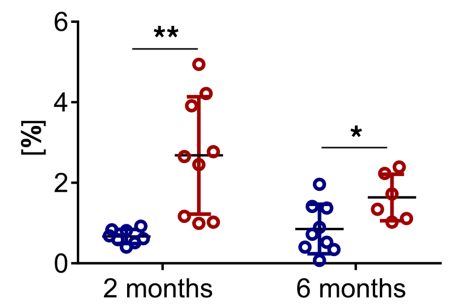

Figure 1. Myelodysplastic NHD13 mice display decreased bone mineralization and high levels of FGF-23. Femora, vertebrae as well as tibiae, and serum of 2-month-old (2M) and 6-month-old (6M) WT (WT) and NUP98/HOXD13 (NHD13) mice were collected for histomorphometric and serum analysis. (A) Representative images of von Kossa/van Gieson-stained vertebra and tartrate-resistant acid phosphatase-stained (TRAP-stained) vertebra of 6-monthold WT and NHD13 mice are depicted using the CellSens program and Microscope Axio Imager M1 (Carl Zeiss). In the TRAP-staining, osteoclasts are stained in red, while arrows indicate osteoblasts. Osteoid is stained in pink by von Kossa/van Gieson. Magnification: whole vertebra 2.5× (scale bars: 200 $\mu \mathrm{m})$; zoom of trabeculae $20 \times$ (scale bars: $50 \mu \mathrm{m}$ ). (B) Quantitative data of trabecular number in TRAP-stained femora $(2 \mathrm{M}, n=6-8 ; 6 \mathrm{M}, n=9-11),(\mathbf{C}-\mathbf{F})$ osteoclasts ( $2 \mathrm{M}, n=5-7 ; 6 \mathrm{M}, n=9-13)$, and osteoblasts per bone perimeter ( $2 \mathrm{M}, n=6-8 ; 6 \mathrm{M}, n=9-13)$ in femora, as well as measurement of bone-resorbing marker C-terminal telopeptide of type 1 collagen (CTX-1) (2M, $n=6-10 ; 6 \mathrm{M}, n=7-10)$ and bone-forming marker procollagen type $1 \mathrm{~N}$-terminal propeptide (P1NP) (2M, $n=7-8 ; 6 \mathrm{M}, n=7-13$ ) in the serum by ELISA. (C) Double calcein labeling was used to assess bone formation rate in tibiae (2M, $n=5-8 ; 6 \mathrm{M}, n$ = 6-9). ( $\mathrm{H}$ and $\mathrm{I})$ Osteoid volume per bone volume (2M, $n=8-9 ; 6 \mathrm{M}, n=6-9)$ and osteoid width (2M, $n=7-10 ; 6 \mathrm{M}, n=6-11)$ were determined in von Kossa/ van Gieson-stained tibiae. (J) C-terminal FGF-23 serum levels were measured by ELISA ( $2 \mathrm{M}, n=5-8 ; 6 \mathrm{M} \mathrm{WT}, n=6-8)$. Data in B-J are shown as mean \pm SD of 3 independent experiments and were analyzed by the 2 -sided Student's $t$ test. ${ }^{*} P<0.05 ;{ }^{*} P<0.01 ;{ }^{* *} P<0.001$ vs. age-matched WT mice. 
mineralization are normal in NHD13 mice. We next measured serum levels of FGF-23, an osteocyte-produced hormone that regulates serum phosphatelevels and bone mineralization(23). Intact FGF-23 (iFGF-23), the active form of the hormone, was unchanged in 6-month-old NHD13 mice compared with WT mice. In line with that, serum phosphate and calcium levels were also normal (Supplemental Figure 2, A-C). Interestingly, serum levels of the C-terminal fragment of FGF-23 (cFGF-23) were increased 2-fold in 6-monthold but not in 2-month-old NHD13 mice (Figure 1J), suggesting that this factor could still contribute to the elevated production of nonmineralized bone matrix in older NHD13 mice.

Blocking FGF-23 restores bone microarchitecture in NHD13 mice. Because cFGF-23 levels were increased in 6-month-old NHD13 mice, we next treated 2-month-old NHD13 mice and their littermate controls with FGF-23 antibodies for 8 weeks to neutralize the iFGF-23 and to improve bone microarchitecture. To verify whether the FGF-23 antibody has similar effects on phosphate and EPO in WT and NHD13 mice as reported $(24,25)$, we injected a single dose into 6-month-old mice. As expected, phosphate and EPO levels were increased in WT mice after 24 hours. In NHD13 mice, FGF-23 antibodies also enhanced phosphate levels, but they did not affect circulating EPO levels (Supplemental Figure 2, D and E).

Sixteen-week-old NHD13 control mice had a normal bone volume per total volume but a reduced number of trabeculae, increased trabecular separation, and thicker trabeculae (Figure 2, A-D). The administration of FGF-23 antibody over 8 weeks did not change the bone volume in either group (Figure 2A). However, it increased the trabecular number and decreased the trabecular separation in NHD13 mice back to WT levels, and it tended to decrease the trabecular thickness (Figure 2, B-D). Blocking FGF-23 fully restored osteoid surface in NHD13 mice back to WT levels. In WT mice, FGF-23 antibody treatment even further reduced the osteoid surface (Figure 2E). In line with this, FGF-23 blockade shortened the mineralization lag time, both in NHD13 and WT mice (Figure 2F). The high systemic bone formation in NHD13 mice, as reflected by P1NP, was reduced to WT levels by the treatment (Figure 2G). Interestingly, the bone formation rate and the number of osteoblasts as measured at the spine was not affected by the FGF-23 antibody treatment (Figure 2, H and I). Osteoclast parameters of histology were neither altered in NHD13 mice nor by the antibody treatment (Figure 2J).

Surprisingly, blocking FGF-23 did not affect serum phosphorus; calcium; parathyroid hormone (PTH); 1,25-dihydroxyvitamin D3 (1,25-[OH]2 D3); and erythropoietin (EPO) in WT and NHD13 mice (Supplemental Table 1) and also did not affect the degree of mineralization (Supplemental Figure 1, A-C). However, bone with low calcium content (CaLow) was decreased, which is in line with the aforementioned reduction of bone formation (decrease in P1NP and restoration of osteoid surface). Furthermore, the antibody treatment reduced cFGF-23 serum levels in both WT and NHD13 mice (Supplemental Table 1). Intact FGF-23 was highly increased in both antibody-treated groups due to the stabilization of the FGF-23/ FGF-23 antibody complex in the serum.

Taken together, while blocking FGF-23 did not rescue all bone formation parameters, it fully restored bone microarchitecture and the extent of osteoid formation in NHD13 mice.

Neutralization of FGF-23 improves hematopoiesis. Because FGF-23 was recently shown to negatively affect erythropoiesis (22), we further examined whether blocking FGF-23 in NHD13 mice could postpone the onset of anemia. After the treatment period, NHD13 control mice showed significantly lower hemoglobin level and fewer RBC in the blood compared with WT controls (Figure 3, A and B). The administration of FGF-23 antibodies did not affect WBC and platelets of NHD13 mice or blood parameters of WT mice (Supplemental Figure 3, A and B). However, it increased hemoglobin levels and the number of RBC in NHD13 mice after the 8-week treatment period (Figure 3, A and B). In line with these findings, NHD13 mice had a 10-fold increased expression of Epo in the kidney, whereas it was reduced by half after FGF-23 antibody treatment. NHD13 mice had low Fgf23 expression in the kidney, which increased after antibody treatment. Expression of the coreceptor Klotho was not altered in NHD13 mice but was stimulated in both NHD13 and WT mice after treatment (Supplemental Figure 3, C-E). Using flow cytometry, we detected an increase in the total number of lineage-negative, sca-1-positive as well as c-kit-positive cells (LSK) in WT bone marrow after FGF-23 antibody administration, but not in NHD13 mice, which - as expected - showed a lower LSK number compared with WT controls (Figure 3C). In addition, bone marrow cells of all groups were used ex vivo for burst-forming unit erythroid (BFU-E) assay. NHD13 mice had a lower erythroid differentiation potential than WT mice, which was improved after FGF-23 antibody treatment (Figure 3D). Thus, blocking FGF-23 not only improves the bone microenvironment, but also anemia, reflecting a potential therapeutic strategy with a dual mode of action to treat patients with MDS. 
A

Bone volume/total volume

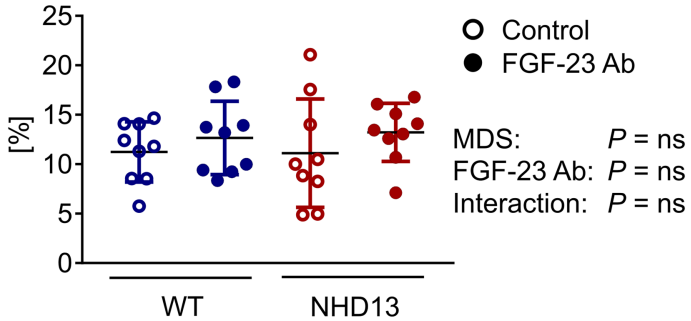

C

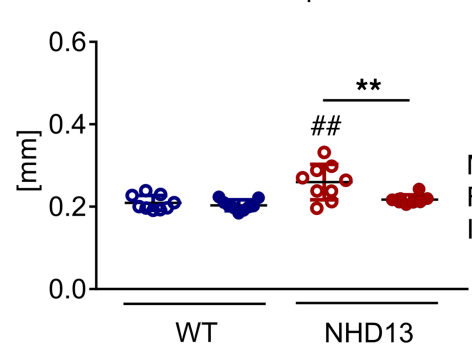

E

Osteoid surface/bone surface

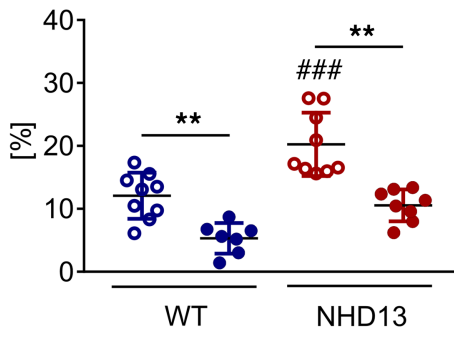

G

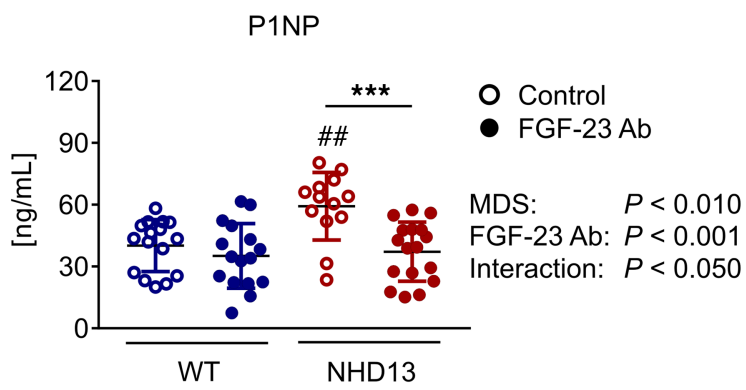

I

Osteoblast number

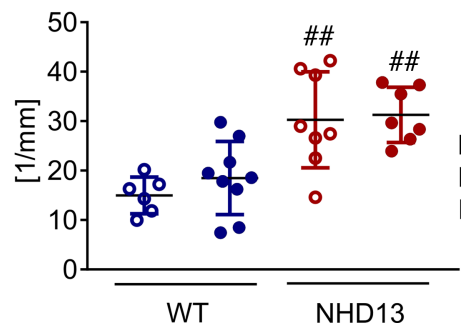

O Control

- FGF-23 Ab

MDS: $\quad P<0.001$

FGF-23 Ab: $P<0.010$

Interaction: $P<0.050$
B

Trabecular number

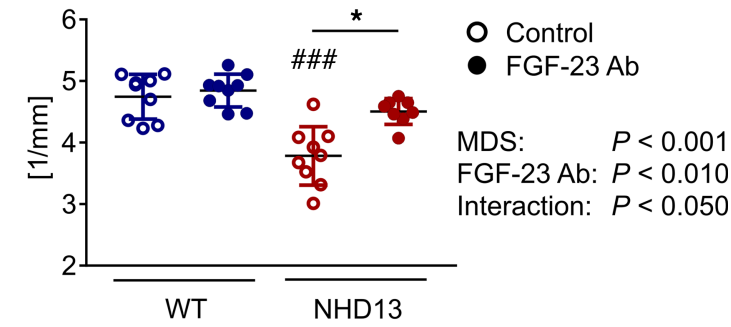

D

Trabecular thickness

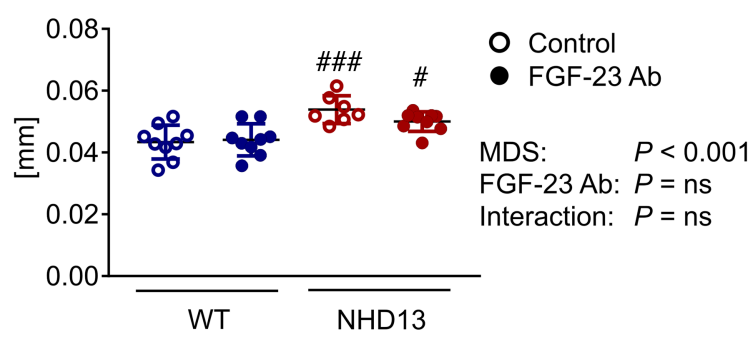

F

Mineralization lag time

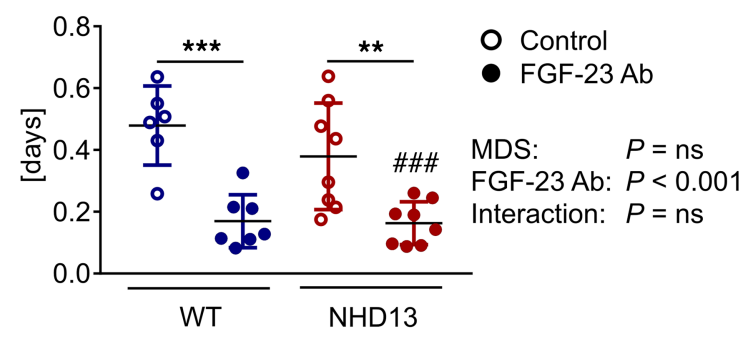

H

Bone formation rate

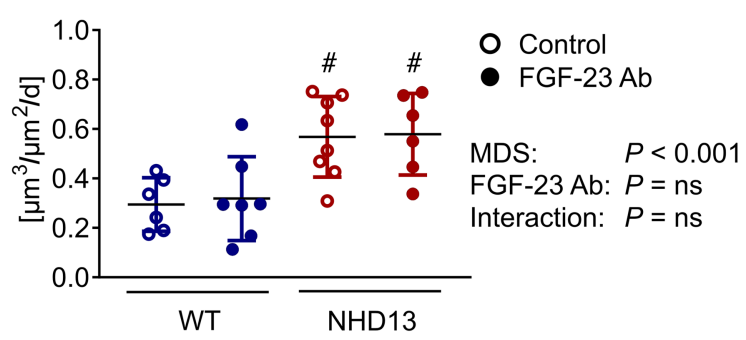

J

Osteoclast number

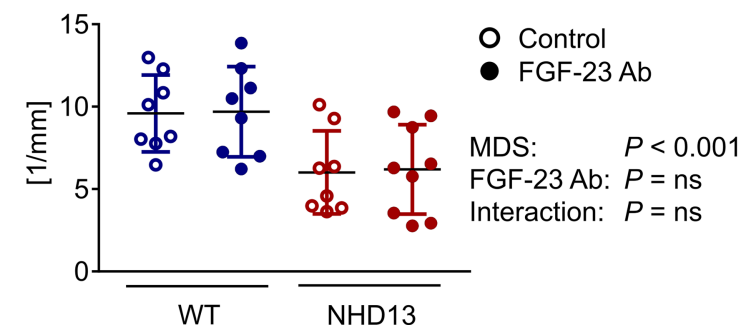

Figure 2. Neutralization of FGF 23 improves bone microarchitecture and mineralization in NHD13 mice. After treatment of 2-month-old WT (WT) and NUP98/HOXD13 (NHD13) mice with FGF-23 Ab over 8 weeks, bone phenotype of femora, bone histology of femora/vertebrae, and serum were analyzed. (A-D) Using $\mu \mathrm{CT}$, quantitative data of bone volume over total volume $(n=9)(\mathbf{A})$, trabecular number $(n=9)(\mathbf{B})$, trabecular separation $(n=$ 9) $(\mathbf{C})$, and trabecular thickness $(n=9)(\mathbf{D})$ were assessed. (E and $\mathbf{F})$ Vertebrae were stained with von Kossa/van Gieson to analyze the osteoid and define the osteoid surface per bone surface $(n=7-9)$ and mineralization lag time $(n=6-8)$. (C) P1NP serum levels were measured by ELISA ( $n=$ 13-16). (H) Five and 2 days before sacrifice, mice received i.p. calcein injections. Based on this double labeling, the bone formation rate in vertebrae 
was determined $(n=6-8)$. (I and J) TRAP-stained femora were used to determine osteoblast $(n=6-9)$ and osteoclast number $(n=8-9)$. Data are shown as mean \pm SD of 5 independent experiments. Statistical analysis was performed by 2 -way ANOVA for the effect of MDS, FGF-23 Ab treatment, and the interaction of both. Statistical significance of Bonferroni's multiple comparisons is denoted. ${ }^{*} P<0.05 ;{ }^{*} P<0.01$; ${ }^{*}{ }^{*} P<0.001$ vs.

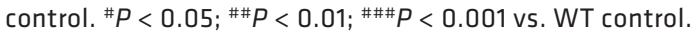

Altered Fgf23 expression in erythroid precursors of NHD13 mice. To analyze which organ contributes to the high FGF-23 serum levels, we first assessed Fgf23 expression in bone of 6-month-old WT and NHD13 mice, since FGF-23 is mainly produced by osteocytes (26). However, we detected a lower skeletal gene expression in NHD13 mice (Supplemental Figure 4). Besides bone, Fgf23 expression has also been reported in other organs such as brain, heart, kidney, and liver (26), but these organs did not show increased Fgf23 mRNA transcripts in NHD13 mice (Supplemental Figure 4). Finally, we assessed Fgf23 mRNA levels in erythroid precursors, as these were recently shown to respond to FGF-23 (22, 27). Even though 6-month-old NHD13 mice had a tendency for lower numbers of Ter119-positive erythroid cells in the bone marrow (Figure 4A), they showed an 8-fold increase in Fgf23 mRNA transcripts (Figure $4 \mathrm{~B})$. However, the expression of $\mathrm{N}$-acetylgalactosaminyltransferase 3 (Galnt3) and family with sequence similarity 20 member C (Fam20c), 2 genes involved in cleavage of FGF-23 (28), were not altered. Furthermore, mRNA levels of all Fgfr (Fgfr1-4) as well as the coreceptor Klotho were increased compared with WT mice (Figure 4B). Finally, when looking more carefully into the Ter119-positive population, we found a higher amount of early erythroid precursor, which may be explainable by the lower apoptosis rate of these cells, but we found a reduced percentage of mature erythrocytes, which showed a higher apoptosis rate (Figure 4, C and D).

As NHD13 mice have highly activated osteoblasts (Figure 1, E and F, and Figure 2, G-I), we examined the possibility that dysplastic erythroid precursors may cause this effect. Thus, we cocultured erythroid precursors of WT and NHD13 mice with WT osteoblasts. Osteoblasts showed a higher expression of Alp and Runx2 when cocultured with erythroid cells from NHD13 mice compared with WT mice (Figure 4E). Moreover, this induction was abolished when FGF-23 neutralizing antibodies were added to the coculture (Figure 4E). These results suggest that the high expression of $F g f 23$ from erythroid precursors is responsible for osteoblast activation.

Patients with MDS have elevated amounts of osteoid and high levels of FGF-23. Finally, we assessed whether our findings in myelodysplastic NHD13 mice are also valid in patients with MDS. Therefore, cFGF-23 and iFGF-23 levels were determined in the plasma of treatment-naive MDS patients of both sexes and all risk groups (Revised International Prognostic Scoring System; IPSS-R). Both cFGF-23 and iFGF-23 levels were increased in women and men with MDS compared with age- and sex-matched patients without hematological disorders (Figure 5, A and B). In addition, correlation analyses were performed for transfusion-independent patients with MDS for FGF-23 and hemoglobin levels, as well as WBC. iFGF-23 did not correlate with hemoglobin levels $(r=0.19 ; P=0.31)$, but with WBC $(r=0.42 ; P<0.05)$. By contrast, we observed a negative correlation between cFGF-23 and hemoglobin levels, but no correlation with WBC (Figure 5, C and D).

To assess whether the increased FGF-23 levels are associated with delayed bone matrix mineralization, we determined the amount of osteoid on bone biopsies from the iliac crest of patients with MDS. According to the literature, healthy individuals have an osteoid volume of $<2 \%$ and osteoid surface of $<20 \%(29,30)$. Regardless of clinical trial involvement or transfusion dependency of MDS patients, osteoid volume per bone volume and osteoid surface per bone surface were increased, but both did not correlate with hemoglobin levels (Figure 5, E-G). In mice, we also observed no correlation with osteoid volume but a negative correlation with osteoid surface (Supplemental Figure 5, A and B). Furthermore, we confirmed high iFGF-23 and cFGF-23 plasma levels in this cohort (compared with controls in Figure 5, A and B, as well as ref. 31), while calcium, phosphate, bone specific alkaline phosphatase, and creatinine were in the normal range (Table 1). Similar to the correlation in mice (Supplemental Figure 5, C-F), iFGF-23 did not correlate with hemoglobin levels $(r=-0.13 ; P=0.56)$, while cFGF-23 was negatively correlated regardless of transfusion dependency (Figure $5 \mathrm{H}$ ). Moreover, cFGF-23 correlated positively with the osteoid surface but not with osteoid volume (Figure 5, I and J). Thus, these data show elevated FGF-23 serum levels in patients with MDS, which may be an important driver of poor bone mineralization and impaired erythropoiesis in MDS 
A

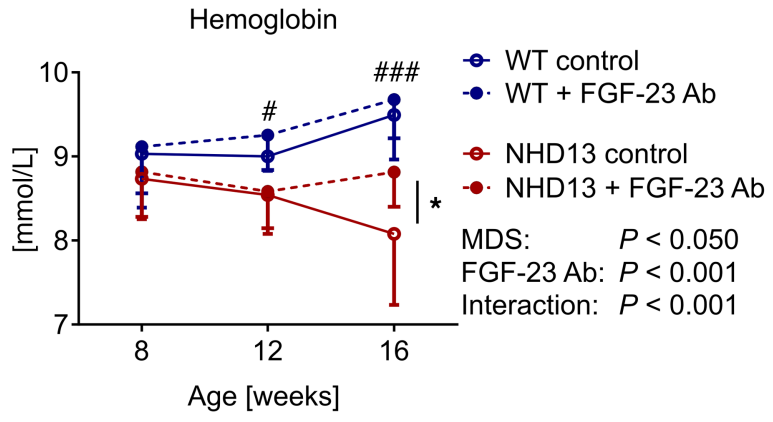

C

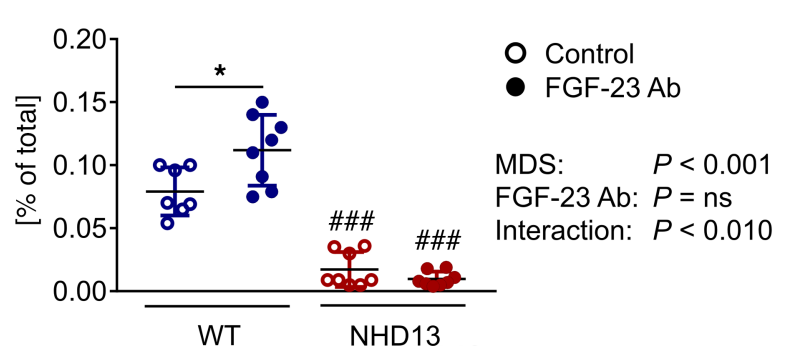

B

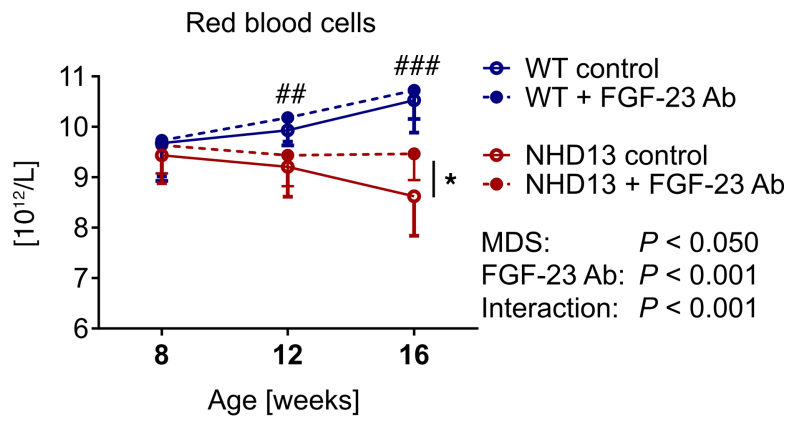

D

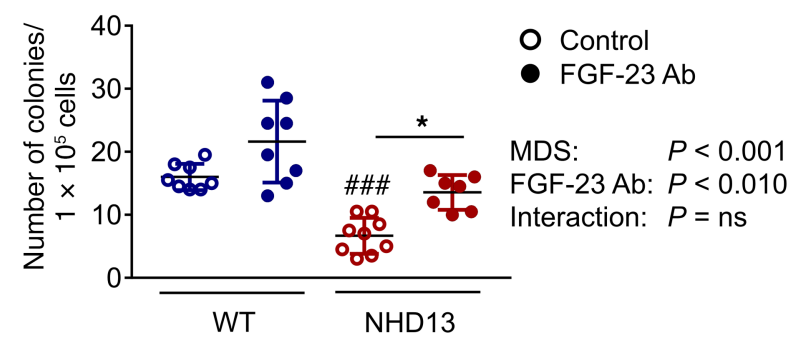

Figure 3. Neutralization of FGF 23 delays the onset of anemia. Eight-week-old NUP98/HOXD13 (NHD13) mice and their littermate WT (WT) mice were treated with FGF-23 neutralizing Ab over 8 weeks. (A and B) Throughout the experiment, retrobulbar blood of the mice was used to analyze blood parameters, hemoglobin levels $(n=17-19)$ and RBC $(n=17-19)$, once a month. Data are shown as means \pm SD of 12 independent experiments. (C and $\mathbf{D})$ Bone marrow cells were used to determine the total number of LSK by flow cytometry $(n=7-8)$ (C) and the number of erythroid colonies using burst-forming unit erythroid assay $(n=7-9)(\mathbf{D})$. Erythroid colonies were counted after 8 days of cultivation. Data are shown as mean \pm SD of 5 independent experiments. Statistical analysis was performed by 2-way ANOVA for the effect of MDS, FGF-23 Ab treatment, and the interaction of both. Statistical significance of Bonferroni's multiple comparisons is denoted. ${ }^{*} P<0.05$ vs. control. ${ }^{\#} P<0.05$; $\# \#<0.01$; ${ }^{\# \# P}<0.001$ vs. WT control.

\section{Discussion}

The majority of patients with MDS develop anemia resulting from insufficient hematopoiesis. Previous studies have highlighted the contribution of the bone microenvironment, in which hematopoiesis occurs, to the progression of MDS. We have also recently shown that the bone microenvironment is impaired in MDS, as patients with MDS have a high prevalence of osteoporosis (8). Thus, in MDS, the physiological interactions between hematopoietic cells and bone cells in the bone microenvironment are disturbed, and a vicious circle develops in which bone cells fail to provide sufficient support for hematopoiesis and dysfunctional blood cells send false signals to distort bone cell activity. Overall, key signals to drive the vicious circle are not well understood; thus, therapeutic options are limited. Here, we provide evidence that FGF-23 negatively affects erythropoiesis, and we propose that targeting FGF-23 may be a potential strategy to treat anemia and bone abnormalities simultaneously in patients with MDS

Our detailed studies on the bones of NHD13 mice showed that their microstructural alterations are mainly derived from a high number of osteoblasts; however, the mineralization lags behind, as indicated by the higher amount of nonmineralized bone (osteoid). This may explain the reduced number of trabeculae, despite a low number of osteoclasts. A similar nonfunctional stage of the osteoblastic lineage in NHD13 mice was also reported by Balderman et al. (11) and by several other studies demonstrating the potential of osteoblast insufficiency and molecular alterations in the bone microenvironment to modulate HSC function $(14,32,33)$. While the osteoid volume was increased 2.5-fold in NHD13 mice, the increase was not as pronounced as in predominant osteomalacia, in which this parameter is increased up to 40-fold (34) and in which the calcium content in the bone is decreased (35). Thus, NHD13 mice do not suffer from overt osteomalacia; however, the high number of osteoblasts also do not seem to mineralize the bone in healthy mice effectively, as the onset of mineralization is delayed.

Bone mineralization is regulated by phosphate homeostasis (36) and 1,25-(OH)2 D3 (37). Recently, FGF-23 has been recognized as a regulator of phosphate homeostasis by stimulating phosphate excretion via effects on the kidney and intestine $(38,39)$, and directly inhibits bone mineralization via suppressing 
A

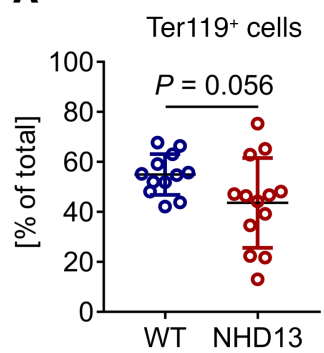

B

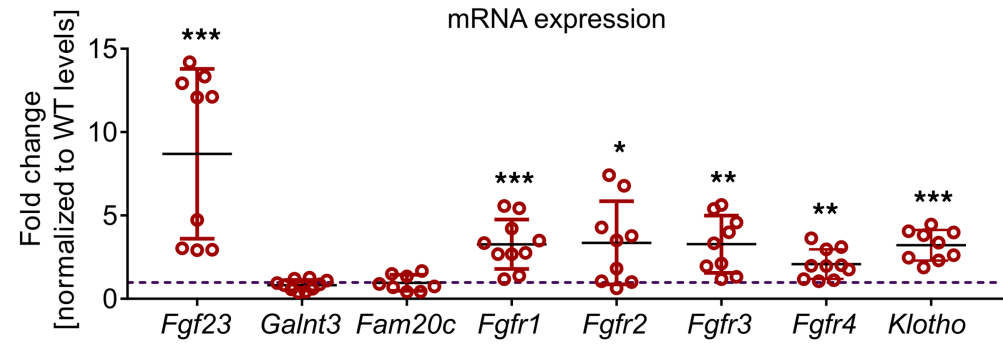

C

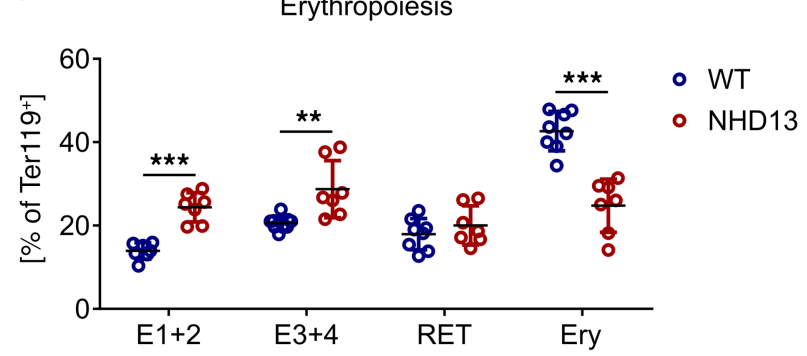

D

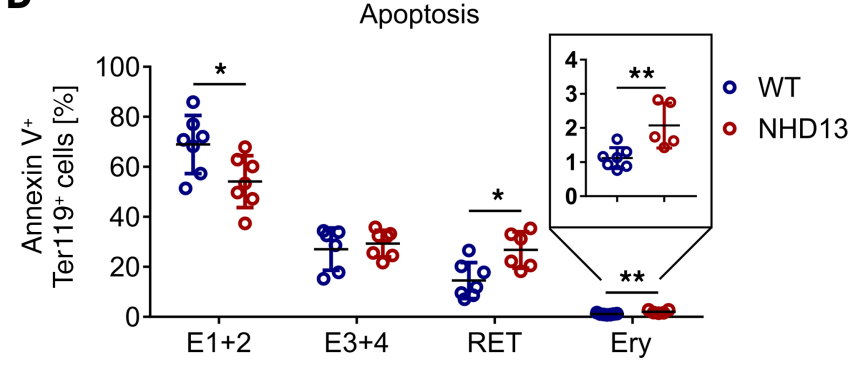

E

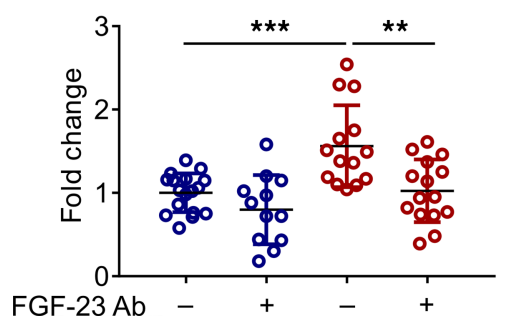

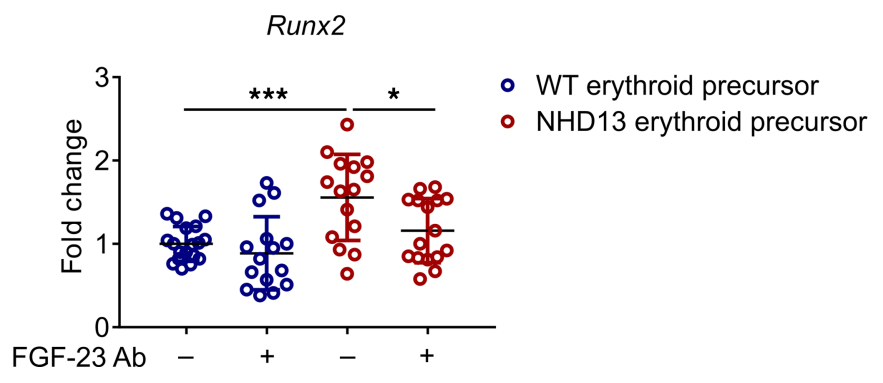

Figure 4. Erythroid precursors of NHD13 mice express high Fgf-23 and activate osteoblasts. (A) Primary bone marrow cells of 6-month-old WT (WT) and NUP98/HOXD13 (NHD13) mice were used to assess the amount of Ter119-positive cells by flow cytometry $(n=12-13)$. (B) In Ter119-positive cells, gene expression of Fgf23, Fgfr1-4, and Klotho were determined by quantitative PCR $(n=8-10)$. (C and D) Erythroid differentiation $(n=7-9)$, as well as apoptosis $(n=5-7)$, in erythroid maturation stages was analyzed using flow cytometry. (E) In addition, erythroid precursors of WT and NHD13 mice were cocultured with WT osteoblast in the presence or absence of FGF-23 antibodies, and the Alp ( $n=12-17)$ as well as Runx2 ( $n=15-18)$ expression was measured in the osteoblasts. Data are shown as mean \pm SD of 3 independent experiments. In B, the dotted line represents WT levels. Statistical analysis was performed by the 2 -sided Student's $t$ test or 1-way ANOVA followed by Bonferroni's multiple comparison. ${ }^{*} P<0.05 ;{ }^{*} P<0.01 ;{ }^{* *} P<0.001$ vs. WT.

osteoblast mineralization $(40,41)$. Interestingly, NHD13 mice had increased osteoid before and after the onset of MDS, while cFGF-23 levels were only increased at the time of developing anemia. Thus, increased cFGF-23 appears rather as a result than a primary cause of disturbed mineralization in NHD13 mice. In line with this, serum phosphate levels were unchanged in NHD13 mice. This suggests that cFGF-23 does not modulate phosphate homeostasis. Indeed, only iFGF-23 leads to phosphate wasting in mice $(42,43)$, and the active hormone was normal in NHD13 mice. Importantly, neutralization of FGF-23 in NHD13 mice still reduced the amount of osteoid, reduced the activity of osteoblasts as indicated by serum P1NP levels, and corrected the bone microarchitecture, while it had no effect on serum phosphate levels. In any case, in addition to hypophosphatemic rickets $(34,44,45)$, which is also characterized by a high amount of osteoid, blocking FGF-23 also appears to be a useful strategy to improve bone mineralization in MDS.

Because previous reports suggest that FGF-23 is a negative regulator of erythropoiesis, we further determined the development of anemia after FGF-23 antibody treatment in NHD13 mice. Indeed, blocking FGF-23 not only promoted mineralization of osteoid, but also improved erythrocyte counts after the 8-week treatment period. In order to identify the source of FGF-23 in NHD13 mice, we analyzed Fgf23 expression in bone, as osteocytes are the main producers of FGF-23 (20). Neither bone nor other tissues reported to express FGF-23, including the brain, kidney, and heart, explained the high serum levels of FGF-23. Surprisingly, we identified a high expression of Fgf23 in erythroid precursors in NHD13 mice, 
A

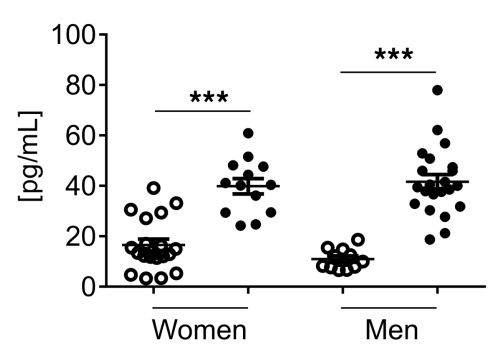

C

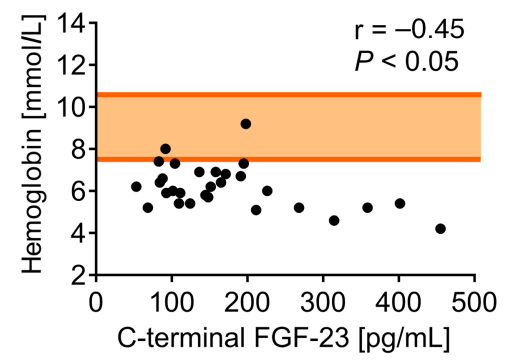

E

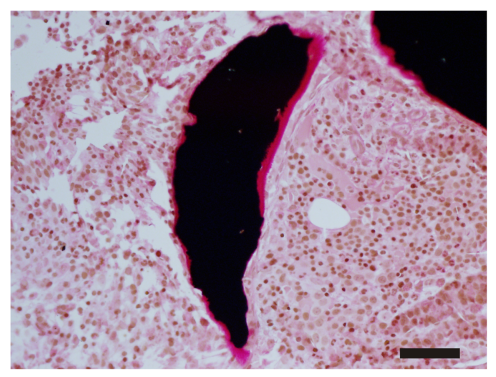

G

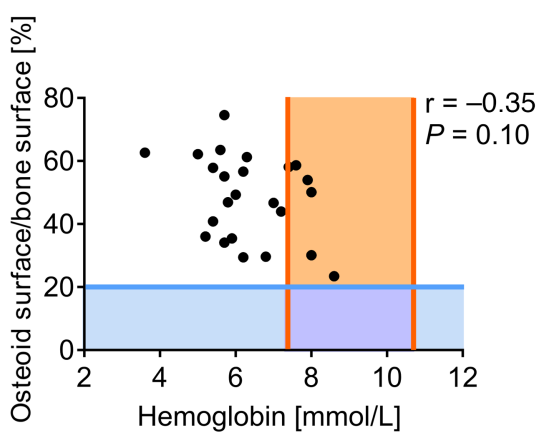

I

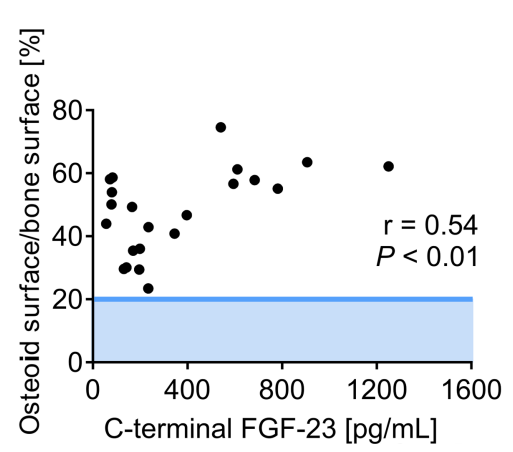

B

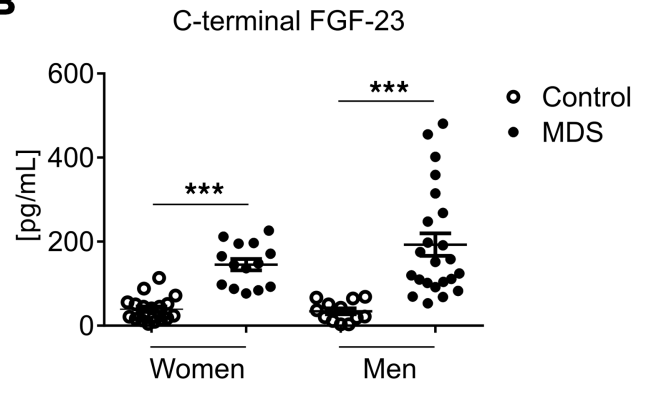

D

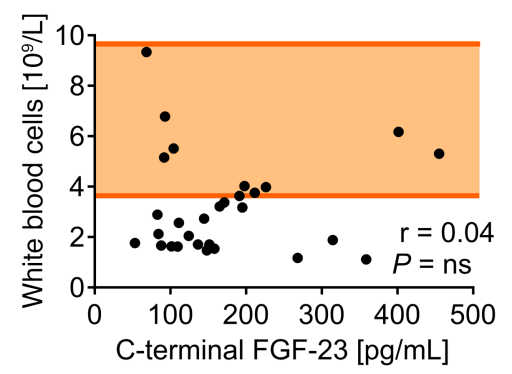

F

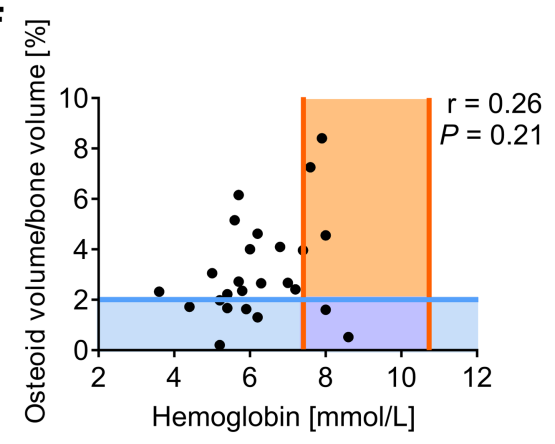

H

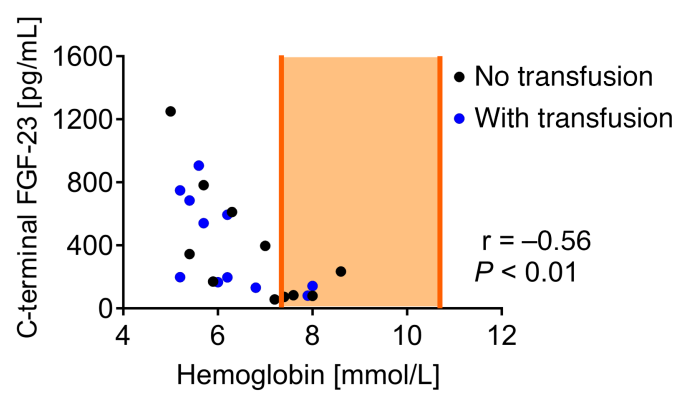

J

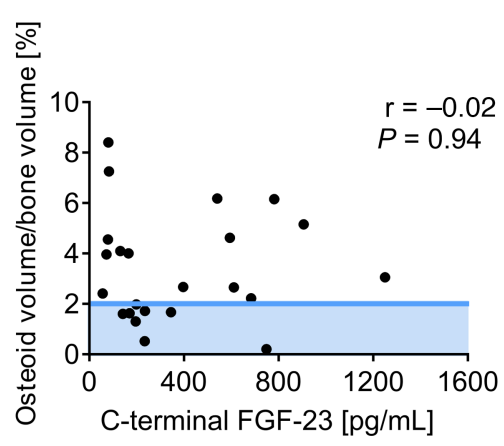

Figure 5. Patients with MDS display high levels of FGF23 and impaired bone mineralization. ( $A$ and $B$ )

Intact (A) and C-terminal (B) FGF-23

serum levels of treatment-naive MDS patients (women, $n=15$; men, $n=23)$ compared with controls (women, $n=20$; men, $n=13$ ) were measured by ELISA. Data are shown as mean \pm SD and were analyzed by the Student's $t$ test. ${ }^{* *} P<0.001$ vs. control. (C and D) Scatter plots and Pearson correlation coefficient $(r)$ of the C-terminal FGF-23 and hemoglobin levels $(n=29)$ (C) or WBC $(n=$ 29) (D). To determine the amount of nonmineralized bone matrix in MDS patients, iliac crest bone biopsies were collected. (E) Representative image of von Kossa/van Giesonstained biopsy is depicted using CellSens program and Microscope Axio Imager M1 (Carl Zeiss). Osteoid is stained in pink. Magnification, $40 \times$ (scale bars: $20 \mu \mathrm{m}$ ). (F-J) Scatter plots and Pearson correlation coefficient $(r)$ were applied to determine the dependence of hemoglobin levels and osteoid volume per bone volume $(n=26)(\mathbf{F})$, osteoid surface per bone surface $(n=24)(\mathbf{C})$, or C-terminal FGF-23 $(n=22)(\mathbf{H})$, as well as the correlation between C-terminal FGF-23 and osteoid surface per bone surface $(n=22)$ (I) or osteoid volume per bone volume $(n=23)(\mathrm{J})$. In all scatter plots, each dot represents an MDS patient. The reference range of healthy individuals for hemoglobin levels or WBC is shown in orange and, for osteoid volume per bone volume or osteoid surface per bone surface, in blue. 
Table 1. Blood, histological, and plasma parameters of MDS patients

\begin{tabular}{|c|c|c|}
\hline & MDS patients $(n=26)$ & Reference values \\
\hline $\begin{array}{l}\text { Red blood cells }[1012 / \mathrm{L}] \\
\text { White blood cells }\left[10^{9} / \mathrm{L}\right] \\
\text { Platelets }\left[10^{9} / \mathrm{L}\right]\end{array}$ & $\begin{array}{l}3.11(2.74-3.61) \\
4.53(2.98-5.87) \\
136(93-276)\end{array}$ & $\begin{array}{c}4.02-5.40 \\
3.80-9.80 \\
150-400\end{array}$ \\
\hline \multicolumn{3}{|l|}{ Histological parameters } \\
\hline \multicolumn{3}{|l|}{ Plasma parameters } \\
\hline $\begin{array}{l}\text { Intact FGF-23 }[\mathrm{pg} / \mathrm{mL}] \\
\text { C-terminal FGF-23 [pg/mL] } \\
\text { CTX-1 [ng/mL] } \\
\text { Osteocalcin }[\mathrm{ng} / \mathrm{mL}] \\
\text { Calcium }[\mathrm{mmol} / \mathrm{L}] \\
\text { Phosphate }[\mathrm{mmol} / \mathrm{L}] \\
\text { BSAP }[\mathrm{U} / \mathrm{L}] \\
\text { Creatinine }[\mathrm{mg} / \mathrm{dL}]\end{array}$ & $\begin{array}{c}39.0(30.0-58.9) \\
235(134-666) \\
0.24(0.16-0.36) \\
7.35(5.56-8.90) \\
2.30(2.23-2.39) \\
1.06(0.91-1.22) \\
30.0(25.2-35.9) \\
85(71-114)\end{array}$ & $\begin{array}{c}24.7 \text { (90\% Cl: } 11.7-48.6)(31) \\
80.6 \text { (90\% Cl: 32.4-136.5) (31) } \\
\text { A (62) } \\
\text { B (63) } \\
2.09-2.54 \\
0.87-1.47 \\
9.4-54.2 \\
44-106\end{array}$ \\
\hline \multicolumn{3}{|c|}{$\begin{array}{l}\text { For continuous variables median (1st-3rd quartile) are given. BV/TV, bone volume per total volume; OV/BV, osteoid volume per bone volume; OS/ } \\
\text { BS, osteoid surface per bone surface; FGF-23, fibroblast growth factor-23; CTX-1, C-terminal telopeptide of type } 1 \text { collagen; BSAP, bone specific } \\
\text { alkaline phosphatase; CI, confidence interval. A, men: } 0.05-0.58 \mathrm{ng} / \mathrm{mL} \text {; postmenopausal women: } 0.09-1.05 \mathrm{ng} / \mathrm{mL} \text {. B, men: 15.4 (12.0-19.4) ng/mL; } \\
\text { postmenopausal women: } 18.6(13.6-25.6) \mathrm{ng} / \mathrm{mL} \text {. }\end{array}$} \\
\hline
\end{tabular}
postmenopausal women: $18.6(13.6-25.6) \mathrm{ng} / \mathrm{mL}$.

along with a high expression of its receptors and the coreceptor Klotho, indicating that these cells may contribute to the elevation of serum FGF-23 levels in NHD13 mice and contribute to alterations in the osteoblast compartment via their production of FGF-23. Future studies will need to clarify this in more detail.

Finally, our study shows that the FGF-23 axis may be relevant in patients with MDS. iFGF-23 and cFGF-23 serum levels were higher in patients with MDS, however, without changes in serum phosphate levels. Moreover, cFGF-23 but not iFGF-23 levels correlated negatively with RBC parameters. Similar to the mouse study, $\mathrm{CFGF}-23$ rather than $\mathrm{iFGF}-23$ may be critical for the interactions with RBC. Comparable increases of cFGF-23 levels were also found in acute myeloid leukemia (46) and in patients with chronic kidney disease and renal anemia (47), which in the latter also correlated negatively with hemoglobin values. Importantly, we collected a unique set of bone biopsies from MDS patients. Our analysis revealed elevated osteoid surface in MDS patients compared with reference cohorts published in literature (29). This parameter correlated positively with cFGF-23. Taken together, in light of the dual effect of FGF-23 inhibition on erythropoiesis and bone mineralization, it appears attractive to target FGF-23 using neutralizing antibodies such as burosumab to concurrently treat MDS to ameliorate anemia and bone abnormalities (48-50).

Wolf et al. have recently classified diseases that are linked to dysregulations of FGF-23 according to their iFGF-23/cFGF-23 ratio (51). For example, in autosomal dominant hypophosphataemic rickets, FGF-23 cleavage is disrupted, leading to a high iFGF-23/cFGF-23 ratio and hypophosphatemia (34, 52). In contrast, under conditions of iron deficiency or inflammation, Fgf 23 expression and cleavage are both increased resulting in a low iFGF-23/cFGF-23 ratio. Since changes in iFGF-23 are minimal, phosphate levels remain unaffected. Finally, in CKD, Fgf23 mRNA transcription is increased in bone cells early in the disease course, likely due to inflammatory signals and iron deficiency, which are both inducers of FGF-23. This leads to normal or high serum phosphate and a high iFGF-23/cFGF-23 ratio $(24,42,45,51,53)$. However, with increasing impairment of kidney function, renal phosphate excretion is reduced, leading to a progressive increase in serum phosphate. In our scenario, MDS likely falls into the iron deficiency/inflammation category, in which cFGF-23 increases but iFGF-23 and phosphate remain constant (42). The iFGF-23/cFGF-23 ratio alterations, however, are likely independent of iron deficiency and inflammation as NHD13 mice are neither iron deficient nor show signs of inflammation. 
Whether cleavage of FGF-23 is affected remains to be investigated. Even though the expression of Galnt3 and Fam20c, which are 2 important posttranslational FGF-23 modifiers, was not altered, their activity could be different, or other FGF-23-modifying enzymes such as furin could be involved.

Our study has potential limitations. Serum phosphate levels were not changed after the 8-week-antibody treatment period. However, serum phosphate was increased after 24 hours of a single injection of the antibody. Thus, we assume that mice adapted to the FGF-23 antibody treatment over time. Previous studies have found similar results showing changes in phosphate, calcium, PTH, and 1,25-(OH)2 D3 levels after 4 weeks of FGF-23 antibody treatment $(34,45)$, while in an additional 2 weeks, these effects subsided (25). With regard to the human cohorts, all MDS patients received therapy at the time of biopsy and some were transfusion dependent. The impact of these drugs on bone and osteoblasts, in particular, is largely unknown (54-56). In mice, high erythropoietin levels are detrimental to bone $(57,58)$. Ideally, bone biopsies should be analyzed from treatment-naive MDS patients to confirm the disturbed bone mineralization. Finally, the low number of patients did not allow for stratification according to MDS subtype and risk group. This, however, could be important to identify patients who would potentially benefit most from an anti-FGF-23 antibody therapy.

In conclusion, our study identifies FGF-23 as a potential driver of anemia and impaired bone mineralization in MDS and suggests that blockade of FGF-23 may be effective to concomitantly improve hematopoiesis and bone health.

\section{Methods}

Patient cohorts. Cohort 1 included plasma samples from untreated MDS patients of both genders (women, $n=15$; men, $n=23$ ), which were used for the first study. The distribution of MDS subtypes and prognosis within the MDS patient cohort are listed in Table 2. As controls, we collected plasma samples from patients with osteoarthritis (women, $n=20$; men, $n=13$ ) from the Department of Orthopedic and Trauma surgery (Technische Universität Dresden, Dresden, Germany). Those patients had no hematological disorders. The number of controls and MDS patients with chronic kidney disease, diabetes mellitus, osteoarthritis, osteoporosis, rheumatoid arthritis, and thyroid diseases are listed in Table 2.

Cohort 2 included bone biopsies that were only available from MDS patients (women, $n=12$; men, $n=$ 14) who were part of the BoHemE study (NCT02867085; clinicaltrials.gov). Blood samples were also collected on this day. The distribution of MDS subtypes and prognosis within the cohort are listed in Table 3. In addition, $14 \mathrm{MDS}$ patients were blood transfusion dependent. After removal of the bone biopsies, they were fixed in $4 \%$ paraformaldehyde (PFA) for 48 hours and embedded into methyl methacrylate (Technovit 9100, Kulzer) and sectioned with a thickness of $4 \mu \mathrm{m}$.

NHD13 mouse model. C57BL/6-Tg(Vav1-NUP98/HOXD13)G2Apla/J (NHD13) mice, which faithfully recapitulate several symptoms of MDS, were used (the Jackson Laboratory). Heterozygous NHD13 (The Jackson Laboratory) and C57BL/6JRj (WT; Janvier Labs) mice were used for breeding. Mice were fed a standard diet as well as water ad libitum, and they were exposed to a 12-hour light/dark cycle in an air-conditioned room at $23^{\circ} \mathrm{C}$. All mice were routinely genotyped.

To analyze bone homeostasis before the onset and after development of MDS, we used 2- and 6-monthold NHD13 male mice and their littermate WT controls. Moreover, 6-month-old WT and NHD13 mice were treated i.p. with a single injection of FGF-23 antibodies $(10 \mathrm{mg} / \mathrm{kg})$ provided by Amgen Inc., diluted in PBS, and sacrificed after 24 hours. Control mice received PBS.

For antibody treatment, 2-month-old NHD13 male mice and their littermate WT controls were treated i.p. 3 times per week over 8 weeks with a FGF-23 neutralizing antibody ( $5 \mathrm{mg} / \mathrm{kg})$. At baseline and after 4 weeks, retrobulbar blood was collected to analyze the effect of FGF-23 antibody on hematopoiesis.

Five and 2 days before sacrifice, all mice received i.p. calcein injections $(20 \mathrm{mg} / \mathrm{kg})$ to assess the bone formation. On the day of sacrifice, retrobulbar blood was taken from the animals. After subsequent dilution with sodium chloride (1:3), the blood parameters were determined using the XN-1000 (Sysmex). Furthermore, serum, long bones, spine, bone marrow, kidney, brain, and heart were collected for structural, histological, and molecular analyses.

Bone microarchitecture. $\mu \mathrm{CT}$ (vivaCT40, Scanco Medical) was performed on excised femora with an isotropic voxel size of $10.5 \mu \mathrm{m}$ (70 kVP; $114 \mu \mathrm{A} ; 200 \mathrm{~ms}$ integration time). We used established protocols from Scanco Medical to evaluate the trabecular bone parameters. For this purpose, 100 slices beneath the growth plate of the femur were analyzed and subsequently visualized. The threshold for bone was set to $220 \mathrm{mg} /$ $\mathrm{HA} / \mathrm{cm}^{3} . \mu \mathrm{CT}$ parameters were reported according to international guidelines (59). 
Table 2. Characteristics of controls and untreated MDS patients

\begin{tabular}{|c|c|c|}
\hline & $\begin{array}{l}\text { Controls } \\
(n=33)\end{array}$ & $\begin{array}{l}\text { MDS patients } \\
(n=38)\end{array}$ \\
\hline \multicolumn{3}{|l|}{ Sex, $n$} \\
\hline Women & 20 & 15 \\
\hline Men & 13 & 23 \\
\hline \multicolumn{3}{|l|}{ Age, years } \\
\hline Women & 76 (69-77) & $69(64-73)$ \\
\hline Men & $69(61-76)$ & $68(63-74)$ \\
\hline \multicolumn{3}{|c|}{ Time since MDS diagnosis, months } \\
\hline Women & & $5(1-17)$ \\
\hline Men & & $2(1-16)$ \\
\hline \multicolumn{3}{|c|}{ WHO 2016 classification, $n$} \\
\hline MDS-SLD & & 2 \\
\hline MDS-MLD & & 17 \\
\hline MDS-RS-SLD & & 1 \\
\hline MDS-RS-MLD & & - \\
\hline MDS with del(5q) & & - \\
\hline MDS-EB1 & & 7 \\
\hline MDS-EB2 & & 11 \\
\hline \multicolumn{3}{|l|}{ IPSS-R classification, $n$} \\
\hline Very low risk & & 2 \\
\hline Low risk & & 6 \\
\hline Intermediate risk & & 10 \\
\hline High risk & & 11 \\
\hline Very high risk & & 9 \\
\hline \multicolumn{3}{|c|}{ Diseases and comorbidities, $n$} \\
\hline Chronic kidney disease & 3 & - \\
\hline Diabetes mellitus & 14 & 4 \\
\hline Osteoarthritis & 33 & - \\
\hline Osteoporosis & 3 & 1 \\
\hline Rheumatoid arthritis & 3 & 1 \\
\hline Thyroid diseases & 5 & 6 \\
\hline
\end{tabular}

Bone histology, histomorphometry, and quantitative backscattered electron microscopy. To investigate the trabecular number and the number of osteoclasts, as well as osteoblasts per bone perimeter, femora fixed in 4\% PFA were used for paraffin histology. TRAP staining was performed on $2 \mu \mathrm{m}$-thick paraffin sections. For the evaluation of bone parameters, an area of $0.36 \mathrm{~mm}^{2}$ beneath the growth plate in the femora and $0.90 \mathrm{~mm}^{2}$ in the middle of vertebrae was analyzed using the Osteomeasure software. Pictures were taken with the CellSens program.

The fourth and fifth vertebral body, tibiae, and human bone biopsies were embedded in methyl methacrylate after fixation in 4\% PFA to perform bone formation analysis and to determine the amount of osteoid. Afterward, 7- $\mu \mathrm{m}$ sections were used for the assessment of the fluorescence labels (calcein) using fluorescence microscopy. The injected calcein is incorporated into the mineralization fronts, and the distance between the labeling lines (mineral apposition rate, MAR) multiplied by the mineral surface per bone surface (MS/BS) provides the bone formation rate per bone surface (BFR/BS). To assess the osteoid volume (OV), osteoid surface (OS), and osteoid width (O.Wi), 4- $\mu \mathrm{m}$ sections were stained with von Kossa/ van Gieson. Furthermore, these parameters were used to calculate the osteoid maturation time (Omt $=$ $\mathrm{O} . \mathrm{Wi} / \mathrm{MAR})$ and mineralization lag time $(\mathrm{MLT}=\mathrm{O} . \mathrm{Wi} /[\mathrm{MAR} \times \mathrm{MS} / \mathrm{OS}])$. The Osteomeasure software was used to analyze an area of $0.90 \mathrm{~mm}^{2}$ for osteoid staining beneath the growth plate of the tibiae. For the vertebral bodies, an area in the middle of $0.72 \mathrm{~mm}^{2}$, as well as $1.44 \mathrm{~mm}^{2}$, was evaluated for calcein labeling and for the evaluation of bone mineralization, respectively. Osteoid pictures were taken using the CellSens 


\section{Sex, $n$}

Women

Age, years

Women

Men

Time since MDS diagnosis, months

$\begin{array}{lr}\text { Women } & 38(14-61)\end{array}$

Men

\section{WHO 2016 classification, $n$}

MDS-SLD

MDS-MLD

MDS-RS-SLD

MDS-RS-MLD

MDS with del(5q)

MDS-EB1

MDS-EB2

\section{1}

6

3

7

1

4

IPSS-R classification, $n$

Very low risk

Low risk

Intermediate risk

High risk

Very high risk

Clinical trial involvement, $n$

Yes

No

Transfusion-dependent, $n$

Yes

No

For continuous variables median (first-third quartile) are given. SLD, single-lineage dysplasia; MLD, multilineage dysplasia; RS, ring sideroblast; EB, excess blasts; IPSS-R, revised international prognostic scoring system.

program, while fluorescence pictures were taken using the AxioVision 4.8 program. The measurements, terminology, and units used for histomorphometric analysis were those recommended by the Nomenclature Committee of the American Society for Bone and Mineral Research (ASBMR) (59).

In addition, methyl methacrylate-embedded tibiae were used for quantitative backscattered electron imaging (qBEI), which was performed as described previously (60). For the determination of the BMDD, the gray levels (corresponding to local calcium concentrations) were analyzed in calibrated backscattered electron images (with $0.88 \mu \mathrm{m} /$ pixel resolution). The following BMDD parameters were determined in metaphyseal bone: weighted mean calcium concentration (CaMean), CaLow (percentage of low mineralized area below the 10th percentile of WT mice reference BMDD), and CaHigh (percentage of high mineralized area below the 90th percentile of WT mice reference BMDD). Of note, qBEI determines the calcium concentrations within the mineralized matrix. The amount of osteoid is not assessed by this technique and, thus, does not affect the calculation of the BMDD parameters.

Plasma and serum analysis. FGFs (Quidel); bone turnover markers and calcium and phosphate (IDS); and bone-specific alkaline phosphatase and creatinine (Roche) were measured in plasma using commercially available ELISA. The corresponding blood parameters (RBC, WBC, and platelets) of MDS patients were determined using the Sysmex XN-1000.

In order to exclude the influences of the diet on the phosphate and calcium levels in the blood of mice, food was removed from the cages 12 hours before sacrifice. Commercially available ELISAs were 
performed to analyze the bone formation marker P1NP, CTX-1, and 1,25-(OH)2 D3 (IDS); EPO (CloudClone Corp.); PTH, cFGF-23, and iFGF-23 (Quidel Corporation); and calcium and phosphate (Bio Vision Incorporated). All assays were performed according to manufacturer's protocols. Parameters were quantified by measuring the absorbance indicated in the protocol using the FLUOstar OMEGA and evaluated with the associated analysis program.

$R N A$ isolation, reverse transcription and quantitative PCR. Total RNA was extracted from bone, heart, brain, and Ter119-positive bone marrow cells; however, 2 different methods were used for the first step for the organs. The long bones and brains were crushed in liquid nitrogen, whereas a disperser was used to homogenize hearts and kidneys. To isolate Ter119-positive cells, primary bone marrow cells were stained with anti-murine biotin-labeled Ter119 (TER-119, Invitrogen), followed by incubation with magnetic Dynabeads biotin binder (Invitrogen). The labeled Ter119-positive cells were separated from the other bone marrow cells using DynaMag-2 magnet (Thermo Fisher Scientific). The crushed organs and Ter119-positive erythroid cells were lysed in TriFast (VWR). RNA isolation was proceeded according to the manufacturer's protocol, and the RNA was quantified by the NanoDrop spectrophotometer (Peqlab). Subsequently, the produced cDNA (M-MLV Reverse Transcriptase, Promega) was used for SYBR green-based real-time PCR reactions according to standard protocol (Applied Biosystems). The murine primer sequences were: $\beta$-actin sense (s): 5' - GATCTGGCACCACACCTTCT - 3', antisense (as): 5' - GGGGTGTTGAAGGTCTCAAA - 3'; Alp s: 5' - CTACTTGTGTGGCGTGAAGG - 3', as: 5' - CTGGTGGCATCTCGTTATCC - 3'; Epo s: 5' - CACCAGAGACCCTTCAGCTT - 3', as: 5' - GTGGTATCTGGAGGCGACAT - 3'; Fam20c s: 5' - ACATGACCAAGGAGATCCG - 3', as: 5' - CAGATGTTGTTGGCTGGAG - 3'; Fgf23 (52) s: 5' - GTGTCAGATTTCAAACTCAG - 3', as: 5' - GGATAGGCTCTAGCAGTG - 3'; Fgfrl (22) s: 5' - TGATGGGAGAGTCCGATAGAG - 3', as: 5' - CCTGAAGACTGCTGGAGTTAAT - 3'; Fgfr2 (22) s: 5' - CCAGCACTGGAGCCTTATTAT - 3', as: 5' - GTGGTTGATGGACCCGTATT - 3'; Fgfr3 (22) s: 5' - CTAAATGCCTCCCACGAAGAT - 3', as: 5' - CTGAGGATGGAGCATCTGTTAC - 3'; Fgfr4 (22) s: 5' - CCTGAGGCCAGATACACAGATA - 3', as: 5' - GGATGACTTGCCGATGATACAC - 3'; Galnt3 s: 5' - TCTTCACCTGCCATACTGCTG - 3', as: 5' - TTCCTTTCTTGCTGCCTGAC - 3'; Hprt (61) s: 5' - TTGCTGACCTGCTGGATTAC - 3', as: 5' - GTTGAGAGATCATCTCCACC - 3'; Klotho s: 5' - TAGACGGGGTTGTAGCCAAG - 3', as: 5' GAGAAGCGAAAGTGGGTGAC - 3'; Runx2 s: 5' - AAATGCCTCCGCTGTTATGAA - 3', as: 5' - GCTCCGGCCCACAAATCT - $3^{\prime}$. Changes in the mRNA level were calculated according to the $\triangle \triangle C T$ method and are presented as $\mathrm{x}$-fold increase relative to $\beta$-actin mRNA level for the organs and to Hprt mRNA level for Ter119-positive cells. All values were normalized to untreated WT mice.

Flow cytometry. Primary murine bone marrow cells were isolated from femora and stained with an antibody cocktail consisting of anti-murine FITC-labeled CD45R (1:150, RA3-6B2, Invitrogen), CD3 (1:200, 17A2, BD Pharmingen), NK1.1 (1:300, PK136, eBioscience), Ter119 (1:400, TER-119, eBioscience), CD11b (1:000, M1/70, BioLegend), CD19 (1:1000, eBio1D3, eBioscience), and GR1 (1:1000, RB6-8C5, BioLegend), as well as anti-murine APC eFluor 780-labeled c-kit (1:200, 2B8, eBioscience) and anti-murine PECy5labeled sca-1 antibody (1:200, D7, eBioscience). To analyze the amount of Ter119-positive cells, primary bone marrow cells were stained with anti-murine PECy7-labeled CD45 (1:200, 30-F11, Invitrogen), anti-murine PE-labeled CD44 (1:200, IM7, eBioscience), and anti-murine FITC-labeled Ter119 (1:100, TER-119, eBioscience). Anti-murine APC-labeled streptavidin conjugate (1:200, Invitrogen) to dectect the anti-murine biotinylated Ter119 antibody (TER-119, Invitrogen), and anti-murine PE-labeled CD44 (1:200, IM7,eBioscience) were used to determine the erythroid differentiation of magnetic isolated Ter119-positive cells. In addition, these cells were stained with anti-murine FITC-labeled annexin V conjugate (1:20, Invitrogen) in the next step. LSK number, erythroid differentiation, and apoptosis rate were measured using the BD LSRII Flow Cytometry Cell Analyzer (BD Biosciences). Data were evaluated using FlowJo V10 software.

$B F U-E$ assay. BFU-E assay was performed using methylcellulose-based medium with recombinant cytokines (MethoCult GF M3434, Stemcell Technologies). Therefore, $2 \times 10^{5}$ primary bone marrow cells were added to $3 \mathrm{~mL}$ of medium and divided on 2 cell culture dishes $\left(35 \mathrm{~cm}^{2}\right)$. After 8 days of incubation, the number of erythroid colonies was counted using a Zeiss Axiovert 40C microscope.

Coculture of erythroid precursors and osteoblasts. For coculture, LSK from 6-month-old WT and NHD13 mice were isolated by lineage depletion with anti-murine biotin-labeled CD45R (1:400, RA3-6B2, eBioscience), CD3 (1:800, 17A2, Invitrogen), CD11b (1:400, M1/70, Invitrogen), GR1 (1:800, RB6-8C5, eBioscience), and Ter119 (1:400, TER-119, Invitrogen), as well as magnetic Dynabeads biotin binder (Invitrogen, Schwerte, Germany), and they were cultivated in IMDM (Thermo Fisher Scientific) supplemented with 
15\% FCS (Sigma-Aldrich), 1\% penicillin/streptomycin (Thermo Fisher Scientific), 2 mM L-glutamine (Sigma-Aldrich), $10 \mathrm{U} / \mathrm{mL}$ EPO (Janssen), $10 \mathrm{ng} / \mathrm{mL}$ stem cell factor (PeproTech), $10 \mu \mathrm{M}$ dexamethasone (Sigma-Aldrich), $200 \mu \mathrm{g} / \mathrm{mL}$ holotransferrin (Sigma-Aldrich), and $10 \mu \mathrm{g} / \mathrm{mL}$ insulin (Lilly), as well as 100 $\mu \mathrm{M} \beta$-mercaptoethanol (Thermo Fisher Scientific); they were treated with or without $20 \mathrm{ng} / \mathrm{mL}$ FGF-23 antibody. After 2 days, half of the medium was removed, IMDM containing 20\% FCS, 1\% penicillin/streptomycin, and $100 \mu \mathrm{M} \beta$-mercaptoethanol was added and cells harvested by carefully scraping. In parallel, osteoblasts were isolated from the bone marrow of 6-month-old WT mice and differentiated with DMEM containing 10\% FCS, $1 \%$ penicillin/streptomycin, $10 \mathrm{mM} \beta$-glycerol phosphate, and $100 \mu \mathrm{M}$ ascorbate phosphate. At day 7 of osteoblast differentiation, harvested erythroid precursors were added to the osteoblasts and cocultured with or without $20 \mathrm{ng} / \mathrm{mL}$ FGF-23 antibody for 3 days.

Statistics. The murine and human results are presented as means $\pm \mathrm{SD}$, medians with first-third quartiles or as dot plots. All murine experiments were repeated at least 3 times, and GraphPad Prism 7 was used to evaluate the statistical analysis. For the comparison of 2 groups, the 2-tailed Student's $t$ test was performed. To analyze the effect of the FGF-23 neutralizing antibodies, in in vivo experiments a 2-way ANOVA and in the cocultures an 1-way ANOVA were used followed by Bonferroni's multiple comparison. The Pearson correlation coefficient was applied to determine the dependence of 2 variables. A $P$ value less than 0.05 was considered significant.

Study approvals. In both study cohorts, MDS patients who fulfilled the criteria according to the WHO classification, were older than 55 years, and had no previous solid tumors were included. The studies were approved by the IRB of Technische Universität Dresden and conducted in accordance with the Declaration of Helsinki. Written informed consent was obtained from all patients. Participants were identified by number, not by name. All animals were housed in the animal facility of Technische Universität Dresden, and all procedures were approved by the IACUC and the Federal state of Saxony.

\section{Author contributions}

HW, LCH, UP, and MR designed the study. HW, UB, FL, MGLC, BMM, PR, SB, and MR performed experiments and analyzed the results. $\mathrm{EB}, \mathrm{CH}, \mathrm{LCH}$, and UP provided patient material. WGR employed by Amgen provided the FGF-23 antibody and helped with study design. HW, LCH, UP, and MR wrote the manuscript, and all authors read and approved it.

\section{Acknowledgments}

This work was supported by grants from the Deutsche Forschungsgemeinschaft (DFG RA1923/12-1; to MR and UP) and SPP $\mu$ BONE (to CH, LCH, and MR) and by a Meddrive Start-up grant from the Medical Faculty of the Technische Universität Dresden to HW.

Address correspondence to: Martina Rauner, Department of Medicine III \& Center for Healthy Aging, Technische Universität Dresden, Fetscherstraße 74, 01307 Dresden, Germany. Phone: 49.351.458.4206; Email: martina.rauner@ukdd.de.

UP's present address is: Medical Clinic and Policlinic 1, Hematology and Cellular Therapy, Leipzig University Hospital, Leipzig, Germany.

1. Bulycheva E, et al. Myelodysplasia is in the niche: novel concepts and emerging therapies. Leukemia. 2015;29(2):259-268.

2. Bejar R, Levine R, Ebert BL. Unraveling the molecular pathophysiology of myelodysplastic syndromes. J Clin Oncol. 2011;29(5):504-515.

3. Abou Zahr A, Bernabe Ramirez C, Wozney J, Prebet T, Zeidan AM. New Insights into the Pathogenesis of MDS and the rational therapeutic opportunities. Expert Rev Hematol. 2016;9(4):377-388.

4. Raaijmakers MH. Myelodysplastic syndromes: revisiting the role of the bone marrow microenvironment in disease pathogenesis Int J Hematol. 2012;95(1):17-25.

5. Geyh S, et al. Insufficient stromal support in MDS results from molecular and functional deficits of mesenchymal stromal cells. Leukemia. 2013;27(9):1841-1851.

6. Morrison SJ, Scadden DT. The bone marrow niche for haematopoietic stem cells. Nature. 2014;505(7483):327-334.

7. Raggatt LJ, Partridge NC. Cellular and molecular mechanisms of bone remodeling. J Biol Chem. 2010;285(33):25103-25108.

8. Weidner H, et al. Myelodysplastic syndromes and bone loss in mice and men. Leukemia. 2017;31(4):1003-1007.

9. Kode A, et al. Leukaemogenesis induced by an activating $\beta$-catenin mutation in osteoblasts. Nature. 2014;506(7487):240-244.

10. Geyh S, et al. Transforming growth factor $\beta 1$-mediated functional inhibition of mesenchymal stromal cells in myelodysplastic 
syndromes and acute myeloid leukemia. Haematologica. 2018;103(9):1462-1471.

11. Balderman SR, et al. Targeting of the bone marrow microenvironment improves outcome in a murine model of myelodysplastic syndrome. Blood. 2016;127(5):616-625

12. Blau O, et al. Chromosomal aberrations in bone marrow mesenchymal stroma cells from patients with myelodysplastic syndrome and acute myeloblastic leukemia. Exp Hematol. 2007;35(2):221-229.

13. Lopez-Villar O, et al. Both expanded and uncultured mesenchymal stem cells from MDS patients are genomically abnormal, showing a specific genetic profile for the 5q- syndrome. Leukemia. 2009;23(4):664-672.

14. Bhagat TD, et al. Epigenetically Aberrant Stroma in MDS Propagates Disease via Wnt/ $\beta$-Catenin Activation. Cancer Res. 2017;77(18):4846-4857.

15. Poon Z, et al. Bone marrow MSCs in MDS: contribution towards dysfunctional hematopoiesis and potential targets for disease response to hypomethylating therapy. Leukemia. 2019;33(6):1487-1500.

16. Corradi G, et al. Mesenchymal stromal cells from myelodysplastic and acute myeloid leukemia patients display in vitro reduced proliferative potential and similar capacity to support leukemia cell survival. Stem Cell Res Ther. 2018;9(1):271

17. Balaian E, et al. Effects of rigosertib on the osteo-hematopoietic niche in myelodysplastic syndromes. Ann Hematol. 2019;98(9):2063-2072.

18. Balaian E, et al. Erythropoietin inhibits osteoblast function in myelodysplastic syndromes via the canonical Wnt pathway. Haematologica. 2018;103(1):61-68.

19. Lin YW, Slape C, Zhang Z, Aplan PD. NUP98-HOXD13 transgenic mice develop a highly penetrant, severe myelodysplastic syndrome that progresses to acute leukemia. Blood. 2005;106(1):287-295.

20. Erben RG. Update on FGF23 and Klotho signaling. Mol Cell Endocrinol. 2016;432:56-65.

21. Liu S, Zhou J, Tang W, Jiang X, Rowe DW, Quarles LD. Pathogenic role of Fgf23 in Hyp mice. Am J Physiol Endocrinol Metab. 2006;291(1):E38-E49.

22. Coe LM, Madathil SV, Casu C, Lanske B, Rivella S, Sitara D. FGF-23 is a negative regulator of prenatal and postnatal erythropoiesis. J Biol Chem. 2014;289(14):9795-9810.

23. Martin A, David V, Quarles LD. Regulation and function of the FGF23/klotho endocrine pathways. Physiol Rev. 2012;92(1):131-155.

24. Agoro R, et al. Inhibition of fibroblast growth factor 23 (FGF23) signaling rescues renal anemia. FASEB J. 2018;32(7):3752-3764

25. Shalhoub V, et al. FGF23 neutralization improves chronic kidney disease-associated hyperparathyroidism yet increases mortality. J Clin Invest. 2012;122(7):2543-2553.

26. Liu S, Guo R, Simpson LG, Xiao ZS, Burnham CE, Quarles LD. Regulation of fibroblastic growth factor 23 expression but not degradation by PHEX. J Biol Chem. 2003;278(39):37419-37426.

27. Toro L, et al. Erythropoietin induces bone marrow and plasma fibroblast growth factor 23 during acute kidney injury. Kidney Int. 2018;93(5):1131-1141.

28. de Las Rivas M, et al. Molecular basis for fibroblast growth factor 23 O-glycosylation by GalNAc-T3. Nat Chem Biol. 2020;16(3):351-360.

29. Priemel M, et al. Bone mineralization defects and vitamin D deficiency: histomorphometric analysis of iliac crest bone biopsies and circulating 25-hydroxyvitamin D in 675 patients. J Bone Miner Res. 2010;25(2):305-312.

30. Bhan A, Qiu S, Rao SD. Bone histomorphometry in the evaluation of osteomalacia. Bone Rep. 2018;8:125-134.

31. Smith ER, Cai MM, McMahon LP, Holt SG. Biological variability of plasma intact and C-terminal FGF23 measurements J Clin Endocrinol Metab. 2012;97(9):3357-3365.

32. Raaijmakers $\mathrm{MH}$, et al. Bone progenitor dysfunction induces myelodysplasia and secondary leukaemia. Nature. 2010;464(7290):852-857.

33. Visnjic D, Kalajzic Z, Rowe DW, Katavic V, Lorenzo J, Aguila HL. Hematopoiesis is severely altered in mice with an induced osteoblast deficiency. Blood. 2004;103(9):3258-3264.

34. Aono Y, et al. Therapeutic effects of anti-FGF23 antibodies in hypophosphatemic rickets/osteomalacia. J Bone Miner Res. 2009;24(11):1879-1888.

35. Murali SK, Roschger P, Zeitz U, Klaushofer K, Andrukhova O, Erben RG. FGF23 Regulates Bone Mineralization in a 1,25(OH)2 D3 and Klotho-Independent Manner. J Bone Miner Res. 2016;31(1):129-142.

36. Feng JQ, Clinkenbeard EL, Yuan B, White KE, Drezner MK. Osteocyte regulation of phosphate homeostasis and bone mineralization underlies the pathophysiology of the heritable disorders of rickets and osteomalacia. Bone. 2013;54(2):213-221.

37. Masuyama R, et al. Dietary phosphorus restriction reverses the impaired bone mineralization in vitamin $\mathrm{D}$ receptor knockout mice. Endocrinology. 2001;142(1):494-497.

38. Sapir-Koren R, Livshits G. Bone mineralization and regulation of phosphate homeostasis. IBMS Bonekey. 2011;8(6):286-300.

39. Goretti Penido M, Alon US. Phosphate homeostasis and its role in bone health. Pediatr Nephrol. 2012;27(11):2039-2048.

40. Graciolli FG, et al. The complexity of chronic kidney disease-mineral and bone disorder across stages of chronic kidney disease. Kidney Int. 2017;91(6):1436-1446.

41. Wang $\mathrm{H}$, et al. Overexpression of fibroblast growth factor 23 suppresses osteoblast differentiation and matrix mineralization in vitro. J Bone Miner Res. 2008;23(6):939-948.

42. Wolf M, White KE. Coupling fibroblast growth factor 23 production and cleavage: iron deficiency, rickets, and kidney disease Curr Opin Nephrol Hypertens. 2014;23(4):411-419.

43. Goetz R, et al. Isolated C-terminal tail of FGF23 alleviates hypophosphatemia by inhibiting FGF23-FGFR-Klotho complex formation. Proc Natl Acad Sci USA. 2010;107(1):407-412.

44. Zhang R, et al. Unique roles of phosphorus in endochondral bone formation and osteocyte maturation. J Bone Miner Res. 2011;26(5):1047-1056

45. Sun N, et al. FGF23 neutralization improves bone quality and osseointegration of titanium implants in chronic kidney disease mice. Sci Rep. 2015;5:8304.

46. Reinert RB, Bixby D, Koenig RJ. Fibroblast Growth Factor 23-Induced Hypophosphatemia in Acute Leukemia. J Endocr Soc. 2018;2(5):437-443. 
47. Tsai MH, Leu JG, Fang YW, Liou HH. High Fibroblast Growth Factor 23 Levels Associated With Low Hemoglobin Levels in Patients With Chronic Kidney Disease Stages 3 and 4. Medicine (Baltimore). 2016;95(11):e3049.

48. Insogna KL, et al. A Randomized, Double-Blind, Placebo-Controlled, Phase 3 Trial Evaluating the Efficacy of Burosumab, an Anti-FGF23 Antibody, in Adults With X-Linked Hypophosphatemia: Week 24 Primary Analysis. J Bone Miner Res. 2018;33(8):1383-1393.

49. Portale AA, et al. Continued Beneficial Effects of Burosumab in Adults with X-Linked Hypophosphatemia: Results from a 24-Week Treatment Continuation Period After a 24-Week Double-Blind Placebo-Controlled Period. Calcif Tissue Int. 2019;105(3):271-284

50. Minisola S, et al. Tumour-induced osteomalacia. Nat Rev Dis Primers. 2017;3:17044.

51. Edmonston D, Wolf M. FGF23 at the crossroads of phosphate, iron economy and erythropoiesis. Nat Rev Nephrol. 2020;16(1):7-19.

52. Liu S, Zhou J, Tang W, Menard R, Feng JQ, Quarles LD. Pathogenic role of Fgf23 in Dmp1-null mice. Am J Physiol Endocrinol Metab. 2008;295(2):E254-E261.

53. Andrukhova O, Schüler C, Bergow C, Petric A, Erben RG. Augmented Fibroblast Growth Factor-23 Secretion in Bone Locally Contributes to Impaired Bone Mineralization in Chronic Kidney Disease in Mice. Front Endocrinol (Lausanne). 2018;9:311.

54. Poloni A, et al. Azacitidine Treatment in High Risk Myelodysplastic Patients in Complete Haematological Remission Reverts Mesenchymal Stem Cells to a Normal Phenotype. Blood. 2014;124(21):1904.

55. Platzbecker U, et al. ACE-536 Increases Hemoglobin and Reduces Transfusion Burden in Patients with Low or Intermediate-1 Risk Myelodysplastic Syndromes (MDS): Preliminary Results from a Phase 2 Study. Blood. 2014;124(21):411.

56. Suragani RN, et al. Transforming growth factor- $\beta$ superfamily ligand trap ACE-536 corrects anemia by promoting late-stage erythropoiesis. Nat Med. 2014;20(4):408-414.

57. Rauner M, et al. Increased EPO Levels Are Associated With Bone Loss in Mice Lacking PHD2 in EPO-Producing Cells. $J$ Bone Miner Res. 2016;31(10):1877-1887.

58. Shiozawa Y, et al. Erythropoietin couples hematopoiesis with bone formation. PLoS One. 2010;5(5):e10853.

59. Dempster DW, et al. Standardized nomenclature, symbols, and units for bone histomorphometry: a 2012 update of the report of the ASBMR Histomorphometry Nomenclature Committee. J Bone Miner Res. 2013;28(1):2-17.

60. Fratzl-Zelman N, et al. CRTAP deficiency leads to abnormally high bone matrix mineralization in a murine model and in children with osteogenesis imperfecta type VII. Bone. 2010;46(3):820-826.

61. Ledesma-Colunga MG, et al. Prolactin blocks the expression of receptor activator of nuclear factor $\mathrm{\kappa B}$ ligand and reduces osteoclastogenesis and bone loss in murine inflammatory arthritis. Arthritis Res Ther. 2017;19(1):93.

62. Michelsen J, et al. Reference intervals for serum concentrations of three bone turnover markers for men and women. Bone. 2013;57(2):399-404

63. Hannemann A, et al. Reference intervals for serum osteocalcin concentrations in adult men and women from the study of health in Pomerania. BMC Endocr Disord. 2013;13:11. 